\section{Ordinal logistic regression models: application in quality of life studies}

\author{
Modelos de regressão logística ordinal: aplicação \\ em estudo sobre qualidade de vida
}

\author{
1 Faculdade de Medicina, \\ Universidade Federal de \\ Minas Gerais, Belo Horizonte \\ Brasil. \\ 2 Departamento de \\ Estatística, Universidade \\ Federal de Minas Gerais, \\ Belo Horizonte, Brasil. \\ Correspondence \\ M. N. S. Abreu \\ Grupo de Pesquisa em \\ Epidemiologia, Faculdade \\ de Medicina, Universidade \\ Federal de Minas Gerais. \\ Av. Alfredo Balena 190, $6 \underline{\text { o }}$ \\ andar, sala 625 , \\ Belo Horizonte, $M G$ \\ 31130-100, Brasil. \\ merynatali@yahoo.com.br
}

\begin{abstract}
Quality of life has been increasingly emphasized in public health research in recent years. Typically, the results of quality of life are measured by means of ordinal scales. In these situations, specific statistical methods are necessary because procedures such as either dichotomization or misinformation on the distribution of the outcome variable may complicate the inferential process. Ordinal logistic regression models are appropriate in many of these situations. This article presents a review of the proportional odds model, partial proportional odds model, continuation ratio model, and stereotype model. The fit, statistical inference, and comparisons between models are illustrated with data from a study on quality of life in 273 patients with schizophrenia. All tested models showed good fit, but the proportional odds or partial proportional odds models proved to be the best choice due to the nature of the data and ease of interpretation of the results. Ordinal logistic models perform differently depending on categorization of outcome, adequacy in relation to assumptions, goodness-of-fit, and parsimony.
\end{abstract}

Logistic Models; Statistical Methods and Procedures; Quality of Life
Mery Natali Silva Abreu 1,2

Arminda Lucia Siqueira 2

Clareci Silva Cardoso 1

Waleska Teixeira Caiaffa 1

\section{Introduction}

Interest in quality of life has increased in recent years, but the theme is still surrounded by controversy. There is a persistent lack of clarity and consistency as to its meaning, measurement, and data analysis. The World Health Organization (WHO) currently adopts a broad definition of quality of life, as "the individuals' perceptions of their position in life, in the context of the culture and values systems in which they live, and in relation to their goals, expectations, standards, and concerns" 1 (p. 551).

The new concepts of quality of life are consistent with the paradigm shifts that have influenced health policies and practices in the last decades. In addition, the morbidity and mortality profile indicates an increase in the prevalence of chronic non-communicable diseases, while therapeutic advances have also led to increased survival in persons with such diseases. As a result, the impact of these diseases and their treatments are evaluated in terms of their influence on quality of life 2 .

Due to the perception that quality of life is an important aspect of health status, physicians and researchers have attempted to transform it into a quantitative measure that can be compared between different populations and even between different diseases 3 .

In recent decades, various instruments, both specific and generic, have emerged for measuring 
quality of life, in addition to the growing interest in the process of cross-cultural adaptation and validation. This outstanding growth in the theme illustrates the efforts focused on the conceptual and methodological maturity of research involving quality of life. In this context, several questions have emerged, such as: how should quality of life be measured or evaluated? What should the design for studies on quality of life look like? How does one investigate factors associated with better quality of life in patients?

Quality of life is generally measured with questionnaires developed by experts from the area. Questions are formulated on specific aspects of the patent's life, and the results are measured principally by means of ordinal scales, which consist of a series of categories with a given order 4 .

For example, the scale Quality of Life in Schizophrenia (QLS-BR) 5,6,7,8 poses the following question to evaluate the patient's level of social activity: "Do you normally go out with other people to have fun?". The possible answers are scored on a Likert-type scale in seven points and correspond to the following options: never, occasionally, sometimes, and frequently. A higher score indicates better quality of life. This result corresponds to an ordinal variable with a single dimension.

This scale has a total of 21 items, and the final score is defined as the mean of these items, varying from 0 to 6 . These scores are divided into three categories in a new ordinal scale: severely compromised quality of life (0-1), moderately compromised quality of life (2-5), and unaltered quality of life (5-6) 7,8. Thus, the categories for the final result of the QLS-BR scale are related to an underlying continuum, which is the score varying from 0 to 6 , and the ordinal variable can be considered a continuous variable with grouped data.

Some problems arise in measuring the evaluation of quality of life. Quality of life scales tend to generate discrete, asymmetrical, and limited distributions. Normally, these scales are treated as continuous due to the extensive number of categories, as with the Medical Outcomes Study 36Item Short Form Health Survey (SF-36) 3, whose scores vary from 0 to 100 (100 indicating "excellent" quality of life). However, traditional analytical methods like the t test and linear regression that assume at least approximate normalcy may not be appropriate, since the distribution is asymmetrical. In addition, for the SF-36 scale, for example, the values end at the score of 100 and often concentrate at this value, which characterizes data asymmetry. It is thus important to consider the ordinal nature originally presented by these variables 9 .
Considering the ordinal nature of scales used to evaluate quality of life and the importance of studies on this theme, we present a review of the methodology for determining the sample size and analysis of factors associated with quality of life by means of ordinal logistic regression models.

\section{Planning quality of life studies}

Any quality of life study should be preceded by good planning, including the choice of instruments and variables, in addition to adequate sample size calculation. The latter is an essential step for obtaining acceptable power to detect differences or effects in the response variable for a given significance level ${ }^{9}$.

Before choosing the formula used to size the sample, one needs to define the measurement that summarizes the study's principal objective, on which the calculation should be based. In studies with a case-control design, or even in cross-sectional studies in which the target event's prevalence is low, the odds ratio (OR) is used as the risk measurement. Whitehead 10 suggests using the OR as a summary measurement, not only for binary response data but also when working with ordinal data.

\section{Odds ratio for ordinal data}

Suppose the target response $(Y)$ on quality of life has $k$ ordered categories ( $\mathrm{Y} j$ with $j=1,2, \ldots, k$ ) and that two groups (A and B) need to be compared. For the category $j$, OR is given by:

$O R_{j}=\frac{\frac{P\left(Y \leq Y_{j} \mid x^{(A)}\right)}{1-P\left(Y \leq Y_{j} \mid x^{(A)}\right)}}{\frac{P\left(Y \leq Y_{j} \mid x^{(B)}\right)}{1-P\left(Y \leq Y_{j} \mid x^{(B)}\right)}}=\frac{\frac{P\left(Y \leq Y_{j} \mid x^{(A)}\right)}{P\left(Y>Y_{j} \mid x^{(A)}\right)}}{\frac{P\left(Y \leq Y_{j} \mid x^{(B)}\right)}{P\left(Y>Y_{j} \mid x^{(B)}\right)}}=\frac{o d d s^{(A)}}{o d d s^{(B)}}$

According to the usual definition, OR is the ratio between two odds, but now odds is defined in terms of cumulative probabilities. For its interpretation, suffice it to recall that the response has been dichotomized, and that the event is to be classified until the category $j$.

If $\mathrm{A}$ and $\mathrm{B}$ represent, respectively, exposure and non-exposure to a risk factor, OR quantifies the odds of an individual in the exposed group being classified up to a given category, compared to the odds of the unexposed group.

In the context of ordinal data, according to the proportional odds assumption, OR is the same for all categories of the response variable 10 . 
Calculation of sample size and power for ordinal data

Whitehead 10 proposes a non-parametric method based on the assumption of a constant OR, which was simplified by Walters et al. ${ }^{9}$, resulting in the formula (2) for calculating the number of subjects per group ( $n$ ) for significance level $a$ and a power of $(1-\beta) 100 \%$, with $\beta$ as the probability of type II error.

$$
n=\frac{6 \times\left[\left(z_{1-\alpha / 2}+z_{1-\beta}\right)^{2} /(\log \mathrm{OR})^{2}\right]}{\left(1-\sum_{i=1}^{k} \bar{\pi}_{j}^{3}\right)}(2)
$$

In (2), OR is given by the expression (1) and $\bar{\pi}_{j}$ is the mean proportion of subjects in category $j$ of the two groups (A and B), that is, $\bar{\pi}_{j}=\left(\pi_{A j}+\pi_{B j}\right) / 2$. The power corresponding to sample size $n$ is calculated as the accumulated probability of the standard normal distribution of the next percentile:

$z_{1-\beta}=\left[(\log \mathrm{OR}) \times \sqrt{\frac{n\left(1-\sum_{i=1}^{k} \bar{\pi}_{i}^{3}\right)}{6}}\right]-z_{1-a / 2}$

\section{Ordinal logistic regression models}

When it is necessary to control possible confounding factors or even when there is a need to take several factors into consideration, special multivariate analysis for ordinal data is the natural alternative. There are various approaches, such as the use of mixed models or another class of models, probit for example, but the ordinal logistic regression models have been widely publicized in the statistical literature $3,4,9,11,12,13,14,15$, $16,17,18,19,20$.

Consider the response variable $Y$ (for example, quality of life score) with $k$ categories coded in $1,2, \ldots, k$ and $\underset{\rightarrow}{x}=\left(x_{1}, x_{2}, \ldots, x_{p}\right)$ the vector of explanatory variables (co-variables). The $k$ categories of $Y$ conditionally to the values of co-variables occur with probabilities $p 1$, $p 2, \ldots, p_{k}$, that is, $p_{j}=\operatorname{Pr}(Y=j \mid \underset{\rightarrow}{x})$ for $j=1,2, \ldots k$. Modeling of ordinal response data can use simple probabilities $\left(p_{j}\right)$ or accumulated probabilities $\left(p_{1}+p_{2}\right),\left(p_{1}+p_{2}+p_{3}\right), \ldots,\left(p_{1}+p_{2}+p_{3}+\ldots+p_{k}\right)$. In the first case, the probability of each category is compared to the probability of a reference category, or each category to the previous category, as in the adjacent categories model. This article will present logistic models with accumulated probabilities.

Table 1 provides a summary of the principal logistic regression models for the response vari- able with or without ordering, with their respective equations and indications for use. Next, we highlight what are considered some important points for the following ordinal logistic regression models: proportional odds model (POM), two versions of the partial proportional odds model, without restrictions (PPOM-UR) and with restrictions (PPOM-R), continuous ratio model (CRM), and stereotype model (SM).

\section{Proportional odds model (POM)}

The proportional odds model (POM), also known as the cumulative logit model, is indicated when an originally continuous response variable is later grouped 4,11

As shown in Table 1, this model compares the probability of a response less than or equal to a given category $(j=1,2, \ldots, k-1)$ to the probability of a response greater than this category. In addition, this model is composed of $k-1$ parallel linear equations. In the particular case of only two categories $(k=2)$, the POM corresponds exactly to the traditional binary logistic regression model (see BM in Table 1).

The model has $(k-1+p)$ parameters. The model's intercept varies for each of the equations and satisfies the condition $\alpha_{1} \leq \alpha_{2} \leq \ldots \leq \alpha_{k-1}$; furthermore, there are $p$ beta coefficients $(\beta)$ whose elements correspond to the effects of the co-variables on the response variable. For a binary explanatory variable, the $\beta$ coefficient represents the logit of the OR of response $Y$ by the association with $\mathrm{x}$, controlled by the other co-variables. Note that $\beta$ does not depend on $j$, meaning that the relationship between $\underset{\rightarrow}{x}$ and $Y$ is independent of the category. This model provides a single OR estimative for all the categories compared, which can be obtained by exponentiation of the $\beta$ coefficient. This estimate is quite convenient in terms of the model's ease of interpretation and parsimony 4 .

This characteristic of the model resulted in the assumption that McCullagh 19 called proportional odds, hence the model's name. This assumption applies to each co-variable included in the model. Thus, in the process of constructing the model, it is always important to verify whether this assumption is met. Testing the homogeneity of the OR generally uses the score test 14 , referred to by Hosmer \& Lemeshow 18 as the parallel regression test, and which can be used to evaluate the model's goodness-of-fit.

When the $Y$ codes are inverted (i.e., $Y_{1}$ is coded as $Y_{k}, Y_{2}$ as $Y_{k-1}$ and so on), only the signal inversion of the regression parameters occurs. This model also displays the property of invariance in relation to combining the response variable cat- 
Information on the principal logistic regression models (with ordinal and non-ordinal categories).

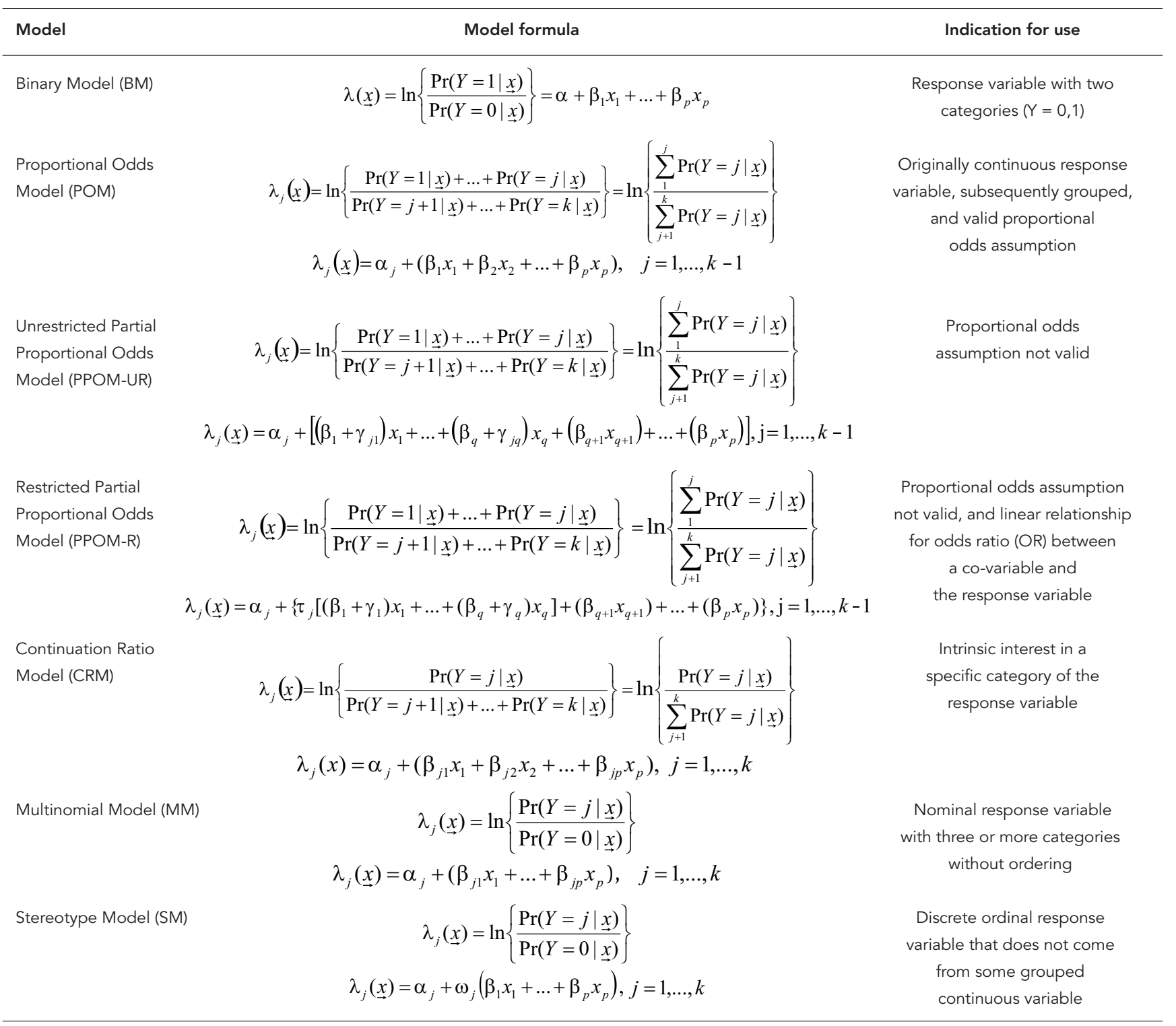

Y: response variable; $\underset{\rightarrow}{x}=\left(x_{1}, x_{2}, \ldots, x_{p}\right)$ : vector of explanatory variables.

egories. This property means that when the $Y$ categories are excluded or regrouped, the co-variables' coefficients ( $\beta$ ) should remain unchanged, although the intercepts $(\alpha)$ are affected.

\section{Partial proportional odds model (PPOM)}

It is rare for all the co-variables included in the model to display the proportional odds property. To contemplate a more realistic situation, the PPOM 21 or partial proportional odds model allows for some co-variables to be modeled with the proportional odds assumption, and for the other variables in which this assumption is not met, specific parameters are included in the model that vary for the different categories that are compared. The PPOM is an extension of the proportional odds model. There are two types of partial proportional odds models, unrestricted and restricted, as follows.

\section{- Unrestricted partial proportional odds model (PPOM-UR)}

As shown in Table 1, according to this model, among the predictive $p$ variables 
$x=\left(x_{1}, x_{2}, \ldots, x_{p}\right)$, only some have proportion$\overrightarrow{\mathrm{al}}$ odds. Without losing generality, let us assume that for the first $q$ co-variables, the proportional odds assumption does not hold true 4 .

For a variable in which the proportional odds property does not hold, say $x_{1}, \alpha_{j}+\beta x_{1}$, it is increased by the coefficient $\gamma_{j 1}$, which is the effect associated with each cumulative logit, adjusted for the other co-variables ${ }^{4}$. Thus, the coefficient of the co-variable is $\alpha_{j}+\beta x_{1}+\gamma_{j 1}$.

For this model, one estimates $(k-1)$ intercepts, $p$ beta coefficients $(\beta)$, which are independent of the categories compared, and $q(k-1)$ gamma parameters $(\gamma)$, which are associated with each co-variable and category in the response variable. If the gamma parameters $(\gamma)$ are null, $\gamma_{j}=0$ for every $j$, the model is reduced to the proportional odds model.

In this model, for the first $q$ co-variables, the angular coefficient depends on $j$, meaning that the relationship between $x$ and $Y$ is dependent on the category. Consequently, ORs are estimated for all the comparisons between response variable categories. For the other co-variables, the angular coefficients $(\beta)$ are independent of $j$, and thus only one OR is estimated.

\section{- Restricted partial proportional odds model (PPOM-R)}

When the relationship between a co-variable and the response variable is not proportional, a kind of tendency is frequently expected. Peterson \& Harrell 21 proposed a model that is applicable when there is a linear relationship between the logit for a co-variable and the response variable 11 .

In this case, restrictions (represented by the gamma parameters and which are fixed scalars) can be inserted as parameters in the model in order to incorporate this linearity (see Table 1).

For a given co-variable, the gamma coefficient $(\gamma)$ does not depend on the cutoff points, but is multiplied by a tau coefficient $(\tau)$ that is specific to each logit 4 .

The choice of the restriction can be decided in various ways. Ideally, it should be determined by using either a data bank from a pilot study or a predefined value.

\section{- Continuation ratio logistic model (CRM)}

Feinberg 22 proposed the continuation ratio logistic model (CRM), which compares the probability of a response equal to a given category, say $Y=j$, to the probability of a higher response, $Y>j$, as shown in Table 1.
For each category $(j=1, \ldots k)$, the model's intercept is $\alpha_{j}$ and the coefficients of the co-variables are the beta coefficients $(\beta)$. This model has different constants and specific coefficients for each comparison. An advantage is that the CRM can be adjusted according to $k$ binary logistic regression models. It is more appropriate when there is intrinsic interest in a specific category of the response variable, and not merely an arbitrary grouping of a continuous variable 11 .

The continuation ratio model is affected by the direction chosen to model the variable, i.e. the property of coding invariance does not hold for this model 16. The OR obtained when modeling increasing severity is not equivalent to the reciprocal obtained when modeling decreasing severity. Thus, one cannot merely invert the coefficient's signal to switch directions in the comparison, as occurs with binary logistic regression models and the proportional odds model 23 .

The assumption of heterogeneity in the cutoff points can be tested by including in the model an interaction term between the target exposure and a factor indicating the cutoff point used in the comparison. The models' goodnessof-fit must be compared with and without the interaction term. If the heterogeneity is significant, the continuation ratio model can be easily adapted with effects for the various cutoff points, using the interaction term included in the model (R Program. The R Foundation for Statistical Computing, Vienna, Austria; http://www. r-project.org).

\section{- Stereotype model}

The stereotype model (SM) should be used when the response variable is intrinsically ordinal and not a discrete version of some continuous variable, as for example with the possible responses to the item from the quality of life scale QLS-BR $5,6,7,8$ (never, rarely, occasionally, sometimes, and frequently), mentioned in the Introduction.

This model was proposed by Anderson 12 who states that medical diagnoses tend to be fixed and invariable, based on the classification of disease severity, such as mild, moderate, and severe. This somewhat stereotypical characteristic is the justification for the model's name. In this case, the model should be flexible enough to capture the natural multidimensionality of these responses 16 .

This is the most flexible model for analyzing ordinal responses and can be considered an extension of the multinomial regression model (see MM in Table 1) 16.

Due to the ordinal nature of the data, a linear structure is imposed on this model's logit. In oth- 
er words, weights are assigned to the coefficients given by $\beta_{j l}=\omega_{j} \beta_{l}$ with $j=1, \ldots, k$ and $l=1, \ldots, p$. (see Table 1$)$. In addition to the weights $\left(\omega_{k}\right)$ for the response variable $Y$, there is a beta parameter for each explanatory variable.

These weights are directly related to the effect of the co-variables. Hence, the OR that is formed will have a tendency towards growth, since the weights are normally constructed by the ordering $\left(0=\omega_{1} \leq \omega_{2} \leq \ldots \leq \omega_{j}\right)$. Thus, the effect of the covariables on the first OR is less than the effect on the second, and so on 4 .

The main difficulty with this modeling is to determine these weights, but some possibilities exist. Greenland 16 suggests that the weights can be decided in advance, in other words, values that are appropriately chosen or estimated, based on data from a pilot study, or using generalized linear models 20 that estimate the weights as additional parameters in the model.

\section{Examples of application}

To exemplify the analysis of quality of life data by means of the above-mentioned models, data were used from a cross-sectional study performed from 1999 to 2005, including 273 patients diagnosed with schizophrenia. These patients are from two mental health referral services from different cities in the State of Minas Gerais, Brazil (Mental Health Referral Service in Divinópolis - SERSAM Divinópolis; and the Mental Health Referral Service in Pampulha - CERSAM Pampulha) 5,6,7,8. Previously trained health professionals conducted the interviews. A questionnaire was applied that included clinical and socio-demographic information on the patients. Quality of life was measured by the QLS-BR, an instrument adapted to and validated for the Brazilian context, presenting good validity and reliability characteristics 5,6.

The QLS-BR structure features a total of 21 items distributed in three specific domains: (1) social, (2) occupational, and (3) intrapsychic and interpersonal relations. The result of the evaluation is scored in a Likert-type scale, with the scores varying from zero to six points and a higher score representing better quality of life. All of the scale's items and domains, in addition to the overall scale, can be analyzed by categorizing the scores, as shown previously 5,6.

Two examples of application were created, and both used two categorical co-variables: gender (female/male) and marital status (married/single), due to their importance in the literature 7,8 associated with quality of life in schizophrenia.
In addition to the descriptive data analysis by means of contingency tables, we tested the association between gender and marital status and quality of life in the patients, by means of the Kruskall-Wallis test using StatXact version 6 (Cytel Inc., Cambridge, USA). Of the four models presented above, in three we used the R Program version 2.2.1. Only the PPOM was adjusted using the Stata software version 9.0 (Stata Corp., College Station, USA).

The proportional odds assumption was tested for each co-variable, and in the final model the score test was used. Each model's fit was evaluated using the deviance test. The goodness-of-fit test 18 was used to compare the multinomial and stereotype models. Finally, the statistical power was calculated using the Whitehead method 10 .

A 5\% significance level was adopted, and the probability of significance was denoted as $\mathrm{p}$.

Example 1: quality of life in the occupational domain of the QLS-BR scale

For this example, quality of life in the occupational domain of the QLS-BR scale was chosen due to its more homogenous distribution among the categories, thus facilitating comparisons. The descriptive data analysis by means of a contingency table showed that the majority of patients (70.8\%) were in the moderately compromised quality of life category, independently of gender or marital status.

Severely compromised quality of life showed a tendency towards worse quality of life for single male patients (39.2\%), but for only two married female patients $(8.3 \%)$. Gender $(p<0.01)$ and marital status $(p=0.04)$ were both significantly associated with low quality of life.

\section{- Proportional odds model}

Univariate and multivariate calculation of the POM was performed, showing that the estimates in the two analyses were quite similar, suggesting minimal confounding. According to Table 2, the parallel regression assumption was not violated ( $p=0.36$ for the score test), indicating OR homogeneity for all the categories compared. Therefore, in this case, there was no need to fit the partial proportional odds models. The deviance test indicates that model is well fitted, and that both variables (gender and marital status) were statistically associated with the outcome. An example of interpretation is that men present twice the odds of worse quality of life category as compared to women.

Table 2 also shows the results of the binary response logistic model after regrouping the mod- 
Results of binary logistic regression, proportional odds, and continuation ratio models using as the response quality of life in the occupational domain of the scale Quality of Life in Schizophrenia (QLS-BR).

\begin{tabular}{|c|c|c|c|c|c|c|}
\hline Type of model & Co-variable (reference) & $\hat{\beta}$ & $\operatorname{SD}(\hat{\beta})$ & $\hat{O R}$ & Wald test & Score test (p) \\
\hline \multirow[t]{2}{*}{ Binary *,** } & Gender (female) & 0.46 & 0.38 & 1.58 & $1.49(p=0.22)$ & - \\
\hline & Marital status (married) & 0.90 & 0.29 & 2.45 & $9.41(p<0.01)$ & \\
\hline \multirow[t]{2}{*}{ Proportional odds *** } & Gender (female) & 0.71 & 0.25 & 2.03 & $2.79(p<0.01)$ & $2.02(p=0.36)$ \\
\hline & Marital status (married) & 0.68 & 0.33 & 1.97 & $2.10(p=0.04)$ & \\
\hline \multirow[t]{2}{*}{ Continuation ratio \# } & Gender (female) & 0.60 & 0.23 & 1.82 & $2.56(p=0.01)$ & $0.18(p=0.86)$ \\
\hline & Marital status (married) & 0.63 & 0.29 & 1.88 & $2.14(p=0.03)$ & \\
\hline
\end{tabular}

Note: the score test is an overall value for the model, i.e., it refers to the co-variables jointly.

SD: standard error; OR: odds ratio.

* The moderately compromised and unaltered quality of life categories were regrouped and compared to severely compromised quality of life;

* Hosmer-Lemeshow test $(p=0.47)$;

** Deviance test $(p=0.55)$;

\# Deviance test $(p=0.26)$.

erately compromised quality of life and unaltered quality of life categories and comparing them to the severely compromised quality of life category. However, in this model, the marital status variable was not significant ( $p>0.05)$, unlike the ordinal model. This shows that the ordinal variable should not be dichotomized, since this can lead to incorrect conclusions, as in this example.

\section{- Continuation ratio model}

Table 2 also shows the results of the CRM without the interaction terms, which were tested and did not prove significant $(\mathrm{p}=0.86)$, showing that the odds ratios are homogeneous, as observed by the proportional odds model. This model also proved adequate according to the deviance test. The OR of 1.88 can be interpreted as the relative odds of single patients having worse quality of life in a specific category, as compared to married patients.

\section{- Multinomial model}

Before adjusting the stereotype model, for a subsequent comparison the multinomial model was fitted and the OR and standard deviations were calculated. In both models, the last category (unaltered quality of life) was considered the reference. Table 3 shows the results.

According to the multivariate analysis, for example, the odds of men showing severely compromised quality of life are 2.46 greater than for women, as compared to unaltered quality of life.

Importantly, in comparisons of moderately compromised versus unaltered quality of life, both co-variables proved non-significant, possibly explained by the proximity between these two categories.

\section{- Stereotype model}

The stereotype model was also fitted and the weights were estimated as model parameters and presented in Table 3. In the multivariate analysis, the marital status variable loses its significance. The odds of men having severely compromised quality of life are three times greater than for women, as compared to unaltered quality of life. The same odds are 1.38 when comparing moderately compromised versus unaltered quality of life.

The goodness-of-fit test shows no significant difference between the multinomial and stereotype models $(p=0.77)$, and both show a good fit ( $\mathrm{p}=0.60$ and $\mathrm{p}=0.58$, respectively).

\section{Example 2: "occupational functioning" item on the QLS-BR scale}

For this example, one of the 21 items from the QLS-BR scale was chosen, the component of the occupational domain called "occupational functioning". Importantly, this was the only item in the scale in which the proportional odds assumption was violated for one of the explanatory variables.

\section{- Proportional odds model}

Table 4 shows the bivariate and multivariate results of the POM for the item "occupational func- 
Results of multinomial logistic regression and stereotype models using as the response quality of life in the occupational domain of the scale Quality of Life in Schizophrenia (QLS-BR).

\begin{tabular}{|c|c|c|c|c|c|c|c|}
\hline \multirow[t]{4}{*}{ Type of model } & \multirow[t]{4}{*}{ Co-variable (reference) } & \multicolumn{6}{|c|}{ Comparisons } \\
\hline & & \multirow{2}{*}{\multicolumn{3}{|c|}{$\begin{array}{l}\text { Unaltered versus severely } \\
\text { compromised quality of life }\end{array}$}} & \multirow{2}{*}{\multicolumn{3}{|c|}{$\begin{array}{l}\text { Unaltered versus moderately } \\
\text { compromised quality of life * }\end{array}$}} \\
\hline & & & & & & & \\
\hline & & $\beta_{1}$ & $\operatorname{SD}\left(\hat{\beta_{1}}\right)$ & $\hat{\mathrm{OR}}$ & $\beta_{2}$ & $\operatorname{SD}\left(\hat{\beta_{2}}\right)$ & $\hat{\mathrm{OR}}_{2}$ \\
\hline \multirow[t]{2}{*}{ Multinomial ** } & Gender (female) & 0.90 & 0.46 & $2.46(p=0.05)$ & 0.02 & 0.41 & $1.02(p=0.97)$ \\
\hline & Marital status (married) & 1.19 & 0.53 & $3.29(p=0.02)$ & 0.71 & 0.45 & $2.03(p=0.12)$ \\
\hline \multirow[t]{2}{*}{ Stereotype $* \star \star$} & Gender (female) & 1.10 & 0.40 & $3.00(p<0.01)$ & 0.32 & 0.40 & $1.38(p<0.01)$ \\
\hline & Marital status (married) & 0.95 & 0.58 & $2.59(p=0.10)$ & 0.28 & 0.58 & $1.32(p=0.10)$ \\
\hline
\end{tabular}

SD: standard error; OR: odds ratio.

* Weight $\omega$ in the stereotype model $=0.29$;

** Deviance test $(p=0.60)$;

$\star \star \star$ Deviance test $(p=0.58)$.

tioning". The parallel regression assumption was violated for the gender co-variable $(\mathrm{p}<0.05$ for the score test), indicating OR heterogeneity in the compared categories. This can also be observed in Table 5, containing the results of the binary logistic regression models with dichotomized quality of life as the response. For the marital status co-variable, the estimated coefficients for the two different comparisons varied only slightly (0.39 and 0.32). On the other hand, confirming the violation of the proportional odds assumption, the coefficients for the gender co-variable vary substantially (1.53 and 0.59 ), such that the OR decreases from 4.64 in the first comparison to only 1.81 in the second. In this case, the POM is not adequate, and the PPOM should be fitted.

\section{- Partial proportional odds model}

Table 5 shows the results of the unrestricted PPOM. Note that two coefficients were estimated for the gender variable (without proportional odds) and only one for the marital status variable.

This model indicates that compared to women, men have nearly five times the odds of severely compromised quality of life as compared to moderately compromised or unaltered quality of life. Meanwhile, compared to women, men have approximately twice the odds of having severely or moderately compromised quality of life versus unaltered quality of life. As for marital status, single as compared to married individuals have 1.44 the odds of worse quality of life.

\section{Calculating statistical power}

Since the study sample used in the example was predetermined, one can calculate the power associated with this sample size $(\mathrm{N}=273)$. Other methodologies have been used 6 , but here the power will be calculated according to Whitehead 10 and Walters et al. 9, since the data used as an example are from a cross-sectional study, and OR was used for the effect measurement.

Marital status was considered the principal co-variable. According to the data from example $1, \bar{\pi}_{1}=0.157 ; \bar{\pi}_{2}=0.291 ; \bar{\pi}_{3}=0.051$, where $\bar{\pi}_{1}$ represents the mean proportion of married and single individuals in the severely compromised quality of life category $\left(Y_{1}\right)$, the mean number of married and single individuals in the moderately compromised quality of life category, and so on $\left(Y_{2}\right)$. Thus, $\sum_{i=1}^{k} \bar{\pi}_{i}^{3}=0.029$. According to the univariate analysis, the OR for the marital status variable is $1.982(\log \mathrm{OR}=0.684)$. Considering a sample size of 136 for each group (approximately half of 273), through expression (3), $z_{1-\beta}=1.249$ and therefore $1-\beta=0.894$, corresponding to a power of nearly $90 \%$ for identification of risk factors for quality of life in the occupational domain.

The data in example 2 produced $z_{1-\beta}=3.160$, thus $1-\beta=0.9992$, corresponding to a power of $99.92 \%$ for identification of factors associated with quality of life in the item "occupational functioning” in the QLS-BR scale.

\section{Discussion}

In general, the ordinal logistic regression models have proven appropriate for analyzing data with 
Results of the proportional odds models using as the response quality of life in the item "occupational functioning" from the scale Quality of Life in Schizophrenia (QLS-BR).

\begin{tabular}{|c|c|c|c|c|c|c|}
\hline Type of analysis & Co-variable (reference) & $\hat{\beta}$ & $\mathrm{SD}(\hat{\beta})$ & $\hat{O R}$ & Wald test & Score test \\
\hline \multirow[t]{2}{*}{ Univariate } & Gender (female) & 1.09 & 0.26 & 2.99 & $4.25(p<0.01)$ & $4.88(p=0.03)$ \\
\hline & Marital status (married) & 0.46 & 0.31 & 1.59 & $1.49(p=0.14)$ & $0.08(p=0.78)$ \\
\hline \multirow[t]{2}{*}{ Multivariate * } & Gender (female) & 1.07 & 0.26 & 2.92 & $4.15(p<0.01)$ & $4.88(p=0.09)$ \\
\hline & Marital status (married) & 0.33 & 0.31 & 1.44 & $1.15(p=0.25)$ & \\
\hline
\end{tabular}

Note: the score test in the multivariate analysis is an overall value for the model, i.e., it refers to the two co-variables jointly.

SD: standard error; OR: odds ratio.

* Deviance test $(p=0.20)$.

Table 5

Results of the binary logistic regression model and unrestricted partial proportional odds model (PPOM-UR) using as the response quality of life in the occupational functioning item from the scale Quality of Life in Schizophrenia (QLS-BR).

\begin{tabular}{|c|c|c|c|c|c|c|c|c|c|}
\hline \multirow[t]{3}{*}{ Type of model } & \multirow{3}{*}{$\begin{array}{l}\text { Co-variable } \\
\text { (reference) }\end{array}$} & \multicolumn{8}{|c|}{ Comparisons } \\
\hline & & \multicolumn{4}{|c|}{$\begin{array}{l}\text { Severely compromised versus moderately } \\
\text { compromised + unaltered quality of life }\end{array}$} & \multicolumn{4}{|c|}{$\begin{array}{l}\text { Severely compromised }+ \text { moderately } \\
\text { compromised versus unaltered quality of life }\end{array}$} \\
\hline & & $\hat{\beta}$ & $\mathrm{SD}(\hat{\beta})$ & OR & Wald test (p) & $\hat{\beta}$ & $\mathrm{SD}(\hat{\beta})$ & OR & Wald test $(p)$ \\
\hline \multirow[t]{2}{*}{ Binary regression } & Gender (female) & 1.53 & 0.36 & 4.64 & $4.28(<0.01)$ & 0.59 & 0.34 & 1.81 & $1.76(0.08)$ \\
\hline & Marital status (married) & 0.39 & 0.41 & 1.48 & $0.95(0.34)$ & 0.32 & 0.41 & 1.38 & $0.79(0.43)$ \\
\hline Unrestricted partial & Gender (female) & 1.54 & 0.36 & 4.65 & $4.29(<0.01)$ & 0.60 & 0.34 & 1.81 & $1.77(0.09)$ \\
\hline proportional odds & Marital status (married) & 0.36 & 0.32 & 1.44 & $1.15(0.25)$ & 0.36 & 0.32 & 1.44 & $1.15(0.25)$ \\
\hline
\end{tabular}

$\mathrm{SD}=$ standard error; $\mathrm{OR}=$ odds ratio.

quality of life measurements as the response. The modeling differs as to the form of these scales: that can have ordered categories grouped on the basis of a continuous underlying variable or discrete categories, but with ordering.

For the data used in the first example, whose response variable was quality of life in the occupational domain, there was no significant difference between the stereotype model and the multinomial model. Nevertheless, the stereotype model is preferable because it considers the ordinal nature of the data and estimates fewer parameters. Importantly, however, this type of model is generally more appropriate for situations in which the ordinal response variable has discrete categories.

However, considering that the response variable used in the example presents ordered categories grouped on the basis of a continuous underlying variable, the proportional odds model or continuation ratio model would be the most recommended. Although these two models agree on the criterion of OR homogeneity for the compared categories, they disagree as to the magnitudes, since they consider different comparisons. The continuation ratio model would not be recommended, although it showed a good fit, since it is indicated when one is interested in the comparison of a specific response category, which is not the case here.

Another relevant point is the fact that comparisons based on various binary logistic regressions can lead to incorrect inferences, as shown in the example analyzed above. Thus, ordinal regression models provide more reliable estimates for analyzing ordinal data. Among the ordinal models, the POM is outstanding due to its parsimony. Considering that its principal restriction - the proportional odds assumption - was not violated for the data in example 1, the POM was considered the most appropriate.

However, for the second example the POM was inadequate due to violation of the assumption. In this case, an alternative ordinal regres- 
sion model is the PPOM. This model provides an interesting option, since the proportional odds assumption does not always hold. The PPOM allows for some variables to have only one OR for all the categories and for others to have an OR for comparisons in each response variable category, as occurred in the second example. One difficulty is that this model is not implemented in many commonly used statistical programs.

Despite some differences in the results of the fitted models, all the fits were reasonable. The POM and PPOM proved adequate for data analysis in the quality of life study on patients with schizophrenia, due to the nature of the response variable for quality of life (grouped continuous variable), in addition to the parsimony and ease of interpretation for the results of these models.

With regard to interpretation of the results of analyses involving ordinal data, one should be certain that it is not done the same as for binary models. Importantly, the interpretation should always take the odds proportionality into account, i.e., the odds of being in a better or worse quality of life category, depending on the case. This procedure is almost always neglected in studies involving ordinal data.

\section{Resumo}

O tema qualidade de vida tem ganhado ênfase nos últimos anos. Tipicamente os resultados da qualidade de vida são mensurados por meio de escalas ordinais. Procedimentos como dicotomizar a variável resposta e desconsiderar a ordenação geram perda de informação e podem ocasionar inferências incorretas. Para análise de dados ordinais, métodos estatísticos específicos são necessários, como modelos de regressão logística ordinal. A proposta deste trabalho é apresentar uma revisão dos modelos de chances proporcionais, de razão contínua, estereótipo e de chances proporcionais parciais. O ajuste, inferência estatística e comparação dos modelos são ilustrados com dados de um estudo sobre qualidade de vida realizado com 273 pacientes com esquizofrenia. Todos os modelos testados mostraram bom ajuste, mas o de chances proporcionais e o de chances proporcionais parciais foram os mais adequados pelo caráter dos dados utilizados e facilidade da interpretação dos resultados. Nem sempre todos os modelos são apropriados, daí a importância de uma escolha cuidadosa, baseada em vários fatores como caráter da variável ordinal, validade dos pressupostos, qualidade do ajuste e parcimônia.

Modelos Logísticos; Métodos e Procedimentos Estatísticos; Qualidade de Vida
It is equally essential that in order to obtain a good statistical analysis, the planning and especially calculation of the sample size should consider the ordinal nature of the quality of life data. Importantly, ordinal models are not the only analytical methods for verifying factors associated with quality of life. There are various other analytical approaches that this article did not discuss, like generalized linear models 20 and decision trees 24 .

Finally, ordinal logistic regression models are appropriate tools for analyzing quality of life data and have proven their great potential for use in other research involving ordinal data. It is recommended to avoid simple procedures, such as dichotomization of the response variable and overlooking ordering, with consequences like loss of information contained in the data and probably incorrect or less appropriate inferences. It is also worth mentioning that there are various types of ordinal variables besides quality of life scores that are used in the public health context and that can also be analyzed by the models discussed in this article.

\section{Contributors}

M. N. S. Abreu designed the project, conducted the literature review and data analysis, and drafted the article. A. L. Siqueira supervised the elaboration and development of the project. W. T. Caiaffa also participated in the project elaboration, literature review, and data analysis. C. S. Cardoso was responsible for the study that resulted in the data bank used as the example and participated in the data analysis. All the authors assisted in drafting the article and approved the final version.

\section{Acknowledgments}

The authors wish to thank the Minas Gerais State Research Foundation (FAPEMIG) for funding the project on Quality of Life in Schizophrenia (grant no. CDS301/02) and the Brazilian National Research Council (CNPq) and Federal Agency for Support and Evaluation of Graduate Education (CAPES) for grants to researchers Waleska Teixeira Caiaffa and Mery Natali Silva Abreu. 


\section{References}

1. Development of the World Health Organization WHOQOL-BREF quality of life assessment. The WHOQOL Group. Psychol Med 1998; 28:551-8.

2. Seidl EMF, Zannon CMLC. Qualidade de vida e saúde: aspectos conceituais e metodológicos. Cad Saúde Pública 2004; 20:580-8.

3. Ciconelli RM, Ferraz MB, Santos W, Meinão I, Quaresma MR. Tradução para a língua portuguesa e validação do questionário genérico de avaliação de qualidade de vida SF-36. Rev Bras Reumatol 1999; 39:143-50.

4. Lall R, Campbell MJ, Walters SJ, Morgan K. A review of ordinal regression models applied on health-related quality of life assessments. Stat Methods Med Res 2002; 11:49-67.

5. Cardoso CS, Bandeira M, Caiaffa WT, Fonseca JOP. Escala de qualidade de vida para pacientes com esquizofrenia (QLS-BR): adaptação transcultural para o Brasil. J Bras Psiquiatr 2002; 51:31-8.

6. Cardoso CS, Bandeira M, Caiaffa WT, Siqueira AL, Fonseca IK, Fonseca JOP. Qualidades psicométricas da escala de qualidade de vida para pacientes com esquizofrenia: escala QLS-BR. J Bras Psiquiatr 2003; 52:211-22.

7. Cardoso CS, Caiaffa WT, Bandeira M, Siqueira AL, Abreu MNS, Fonseca JOP. Factors associated with low quality of life in schizophrenia. Cad Saúde Pública 2005; 21:1338-48.

8. Cardoso CS, Caiaffa WT, Bandeira M, Siqueira AL, Abreu MNS, Fonseca JOP. Qualidade de vida e dimensão ocupacional na esquizofrenia: uma comparação por sexo. Cad Saúde Pública 2006; 22:1303-14.

9. Walters SJ, Campbell MJ, Lall R. Design and analysis of trials with quality of life as an outcome: a practical guide. J Biopharm Stat 2001; 11:155-76.

10. Whitehead J. Sample size calculations for ordered categorical data. Stat Med 1993; 12:2257-71.

11. Ananth CV, Kleinbaum DG. Regression models for ordinal responses: a review of methods and applications. Int J Epidemiol 1997; 26:1323-33.
12. Anderson JA. Regression and ordered categorical variables. J R Stat Soc Ser B Methodol 1984; 46: 1-30.

13. Bender R, Grouven U. Ordinal logistic regression in medical research. J R Coll Physicians Lond 1997; 31:546-51.

14. Brant R. Assessing proportionality in the proportional odds model for ordinal logistic regression. Biometrics 1990; 46:1171-8.

15. Fleck MPA, Leal OF, Louzada S, Xavier M, Chachamovich E, Vieira G, et al. Desenvolvimento da versão em português do instrumento de avaliação da qualidade de vida da OMS (WHOQOL-100). Rev Bras Psiquiatr 1999; 21: 19-28.

16. Greenland S. Alternative models for ordinal logistic regression. Stat Med 1994; 13:1665-77.

17. Hendrickx J. Special restrictions in multinomial logistic regression. Stata Technical Bulletin 2000; 56:18-26.

18. Hosmer DW, Lemeshow S. Applied Logistic Regression. 2nd Ed. New York: John Wiley and Sons; 2000.

19. McCullagh P. Regression models for ordinal data. J R Stat Soc Ser B Methodol 1980; 42:109-42.

20. McCullagh P, Nelder JA. Generalized linear models. London/New York: Chapman and Hall; 1983.

21. Peterson BL, Hanrrel FE. Partial proportional odds models for ordinal response variables. Appl Stat 1990; 39:205-17.

22. Fienberg SE. The analysis of cross-classified categorical data. Cambridge: MIT Press; 1980.

23. Scott SC, Goldberg MS, Mayo NE. Statistical assessment of ordinal outcomes in comparative studies. J Clin Epidemiol 1997; 50:45-55.

24. Breiman L, Friedman JH, Olshen RA, Stone CJ. Classification and regression trees. Pacific Grove: Wadsworth and Brooks; 1984.

Submitted on $21 /$ Jun/2007

Final version resubmitted on 01/Apr/2008

Approved on 08/Apr/2008 